\title{
Abdominal ultrasonographic findings at diagnosis of osteosarcoma in dogs and association with treatment outcome
}

\author{
O. Sacornrattana, N. G. Dervisis and E. A. McNiel \\ Department of Small Animal Clinical Sciences, Center for Comparative Oncology, College of Veterinary \\ Medicine, Michigan State University, East Lansing, MI, 48824, USA
}

Keywords

abdominal ultrasound, canine, metastasis, osteosarcoma
Correspondence address: E. A. McNiel

Tufts Medical Center, 800 Washington St. \# 5609, Molecular Oncology Research Institute, Boston, MA 02111

e-mail:

elizabeth.mcniel@tufts.edu

\begin{abstract}
The purpose of this study was to describe abdominal ultrasonographic findings present at diagnosis of osteosarcoma (OSA) in dogs and to investigate for associations with treatment outcome. Medical records from 118 dogs diagnosed with OSA that had abdominal ultrasonography performed as part of their initial evaluation were reviewed. Fifty-seven percent had ultrasonographic abnormalities identified. The organ with the highest frequency of ultrasonographic changes was the spleen. While most sonographic changes were considered to be either benign or of unknown clinical consequences, metastases were identified in three dogs (2.5\%), two of which (1.7\%) did not have other evidence of metastasis. Dogs with any ultrasonographic abnormality were less likely to receive definitive therapy $(P=0.005)$ and exhibited shorter survival, although the latter observation was not statistically significant $(P=0.071)$. However, the identification of lesions in either the liver $(P=0.021)$ or the kidney $(P=0.003)$ was statistically associated with shorter survival.
\end{abstract}

\section{Introduction}

Osteosarcoma (OSA) is relatively common in dogs and accounts for $85 \%$ of bone malignancies in this species. ${ }^{1-3}$ Although less than $15 \%$ of affected dogs have radiographically detectable pulmonary or osseous metastasis at presentation, approximately $90 \%$ will die from metastatic disease. ${ }^{1,2,4,5}$ Metastasis via the hematogenous route is most common; however, on rare occasions metastatic spread to regional lymph nodes may occur. ${ }^{1,5}$ The most common metastatic sites are lung and bone. ${ }^{1,2}$ With adjuvant chemotherapy, bone and soft tissue are increasingly identified as sites of OSA metastasis, but that typically occurs late in the course of the disease. ${ }^{1,2}$

Abdominal ultrasonography (AUS) is often performed in the initial evaluation of dogs with cancer including those with bone tumours. However, there are no data regarding the utility of AUS when used as a screening tool in the newly diagnosed patient. Therefore, the goal of this study was to describe the frequency and types of AUS findings at the time of diagnosis of osteosarcoma in dogs and to investigate the influence of AUS findings on case outcome.

\section{Materials and methods}

Medical records from the Michigan State University Veterinary Teaching Hospital were reviewed to identify dogs diagnosed with OSA between 1 January 2004 and 31 December 2009. Dogs with cytologically- or histologically confirmed OSA, as well as dogs with radiographic lesions consistent with a diagnosis of OSA were included in the study. Patients were included only if AUS was performed as part of the initial evaluation, with AUS and diagnosis occurring within the same hospitalization episode. Dogs with extraskeletal osteosarcoma and dogs that did not receive AUS at the time of initial staging were excluded. 
Signalment, site of primary tumour (appendicular versus axial), serum alkaline phosphatase (SAP), method of diagnosis, type and number of treatments, ultrasonographic findings, cytological diagnosis of ultrasonographic findings, thoracic radiographic findings and clinical outcome were recorded and analysed. All chest radiographs were interpreted by board-certified radiologists. All abdominal ultrasounds were performed by boardcertified radiologists at Michigan State University Veterinary Teaching Hospital.

\section{Ultrasonographic changes}

An ultrasonographic change was defined as any alteration reported by the radiologist performing the exam. Findings were categorized into the following categories: change in organ size, alteration in echotexture, mass or nodular effect, alteration in architecture of organs, such as layering, cyst formation and presentation of substances in the lumen. Ultrasonographic findings were also categorized based on the organ involved.

\section{Statistical analysis}

Frequencies were reported for categorical variables. For continuous variables, the Shapiro-Wilk test was used to test for normality and the mean and SD was reported.

To investigate for an association between ultrasonographic findings and treatment decisions, a $\chi^{2}$ test was used. For this analysis, patients were categorized as receiving no treatment, palliative treatment or definitive treatment. Definitive treatment was defined as the administration of surgery or definitive radiation therapy in conjunction with systemic chemotherapy of any type. Palliative therapy was defined as the administration of any single treatment modality (surgery, radiation therapy or chemotherapy) alone or in combination with palliative radiation therapy. Untreated patients were those that did not have therapy to specifically target the cancer. Administration of analgesic agents, for instance, was not considered specific cancer treatment. The palliative radiation protocol comprised of three 8-Gy fractions (total dose = 24 Gy) delivered at weekly intervals. The definitive radiation protocol consisted of 18 three-Gy fractions (total dose $=54$ Gy), delivered Monday through Friday. All radiation therapy patients were treated with dual photon energy ( 6 and $10 \mathrm{MV}$ ) linear accelerator.

To investigate the effects of AUS findings on case outcome, survival analytic techniques were used. Survival time was defined as the interval between diagnosis of OSA and death due to the cancer. Cases that were lost to follow-up, dead due to another disease and alive at the time of data collection were censored in the analysis. The impact of signalment variables (age, sex, breed), clinical variables [SAP, tumour site (appendicular versus axial), stage], treatment (definitive versus palliative treatment) and various sonographic changes on survival was evaluated using the Cox proportional hazards regression model. Survival curves were generated using the Kaplan-Meier method. Commercially available software was used for all statistical analyses (PASW Statistics 18.0, IBM ${ }^{\circledR}$ SPSS $^{\circledR}$ Statistics, Armonk, New York, United States). For statistical analyses significance was set at $P<0.05$.

\section{Results}

During 2004 through 2009, 157 dogs were diagnosed either presumptively or definitively with osteosarcoma. One hundred and eighteen dogs (75\%) met the inclusion criteria of this study. The age at diagnosis ranged between 1.4 and 16 years with a mean of $8.2 \pm 2.6$ years. Fifty-three (45\%) were neutered males, 58 (49\%) were spayed females, $4(3 \%)$ were intact males and $3(3 \%)$ were intact females.

There were $33(28 \%)$ mixed breed dogs and $85(72 \%)$ purebred dogs. The most common purebreds were the Labrador retriever $(n=19 / 118$; $16 \%)$ and golden retriever $(n=18 / 118 ; 15 \%)$. Other less frequently represented purebreds were the Rottweiler ( $n=10 / 118 ; 8 \%)$, Greyhound ( $n=$ $6 / 118 ; 5 \%)$, St. Bernard ( $n=4 / 118 ; 3 \%)$, German Shepherd $(n=4 / 118 ; 3 \%)$, Doberman Pinscher $(n$ $=3 / 118 ; 3 \%)$, Newfoundland $(n=3 / 118 ; 3 \%)$, German Shorthair Pointer $(n=2 / 118 ; 2 \%)$, Great Dane $(n=2 / 118 ; 2 \%)$, Irish Wolfhound $(n=$ $2 / 118 ; 2 \%)$ and Siberian Husky $(n=2 / 118 ; 2 \%)$. Other purebreds with a frequency of one were Samoyed, Pomeranian, English Mastiff, American 
Pittbull Terrier, Cane Corso, Collie, Boxer, Basset Hound, Belgian Shepherd and Borzoi.

There were 99 (84\%) appendicular OSA and 19 (16\%) axial OSA. The most common sites of the primary tumour were proximal humerus $(n=31$; $26 \%)$ and distal radius $(n=32 ; 27 \%)$. Other sites were distal femur $(n=12 ; 10 \%)$, mandible $(n=$ $8 ; 6.8 \%)$, distal tibia $(n=6 ; 5.1 \%)$, maxilla $(n=$ $4 ; 3.3 \%)$, proximal femur $(n=3 ; 2.5 \%)$, proximal tibia $(n=3 ; 2.5 \%)$, ulna $(n=3 ; 2.5 \%)$, zygomatic arch $(n=3 ; 2.5 \%)$, mid femur $(n=2 ; 1.7 \%)$, nasal cavity $(n=2 ; 1.7 \%)$, ribs $(n=1 ; 0.8 \%)$, metatarsus $(n=1 ; 0.8 \%)$, ilium $(n=1 ; 0.8 \%)$ and scapula $(n=$ $1 ; 0.8 \%)$. One dog had lesions on both right distal radius and ulna. Another dog had boney changes on both left and right distal radius. In three dogs, the site of the primary tumour was poorly documented as right hind limb, right shoulder and left shoulder, respectively.

The diagnosis of OSA was obtained by fine needle aspiration cytology $(n=39 / 118 ; 33 \%)$ and biopsy $(n=73 / 118 ; 62 \%)$. Six dogs $(n=6 / 118 ; 5 \%)$ had radiographic and clinical presentation consistent with OSA and the diagnosis was presumptive.

One hundred and sixteen dogs $(n=116 / 118$; $98 \%)$ had three-view thoracic radiographs performed at the time of diagnosis. Seven dogs $(n=$ 7/116; 6\%) had evidence of pulmonary metastasis.

Sixty-seven patients $(n=67 / 118 ; 57 \%)$ had ultrasonographic abnormalities identified. A total of 108 ultrasonographic changes were recorded. Thirty-eight lesions ( $n=38 / 108 ; 35 \%)$ were evaluated further cytologically with fine needle aspiration. The spleen was the most commonly affected organ accounting for 31 of 108 (29\%) changes. Other reported organ changes involved the liver $(n=25 / 108 ; 23 \%)$, adrenal glands $(n=16 / 108$; $15 \%)$, lymph nodes ( $n=13 / 108 ; 12 \%)$, kidneys $(n=$ $12 / 108 ; 11 \%)$ and urinary bladder $(n=5 / 108 ; 5 \%)$. Stomach, colon, ovary, uterus, peritoneum and prostate gland each had one sonographic alteration reported.

The ultrasonographic alterations that were observed are described in Table 1. The most common type of sonographic alteration observed was a change in organ size $(n=36 / 108 ; 33 \%)$, most frequently involving the adrenal glands $(n=$ $13 / 108 ; 12 \%)$ and lymph nodes $(n=13 / 108 ; 12 \%)$.
Table 1. Types and frequency of abdominal sonographic alterations identified in dog with osteosarcoma

\begin{tabular}{|c|c|}
\hline Type of changes & $\begin{array}{c}\text { Frequency } \\
(n=108)\end{array}$ \\
\hline Organ size & $36(33 \%)$ \\
\hline Adrenomagaly & 13 \\
\hline Lymphadenopathy & 13 \\
\hline Splenomegaly & 3 \\
\hline Microhepatica & 2 \\
\hline Small adrenal gland & 2 \\
\hline Hepatomegaly & 2 \\
\hline Prostatomegaly & 1 \\
\hline Echogenicity & $23(21 \%)$ \\
\hline Heterogenous spleen & 6 \\
\hline Hyperechoic renal cortices & 6 \\
\hline Heterogenous liver & 3 \\
\hline Hyperechoic liver & 3 \\
\hline Hypoechoic area in the spleen & 2 \\
\hline Hypoechoic liver & 2 \\
\hline Hyperechoic area in the spleen & 1 \\
\hline Nodular/Mass lesions & $34(31 \%)$ \\
\hline Splenic nodules & 16 \\
\hline Liver nodules & 12 \\
\hline Splenic mass & 3 \\
\hline Kidney mass & 1 \\
\hline Mass adjacent to the bladder & 1 \\
\hline Adrenal mass invading caudal vena cava & 1 \\
\hline Architecture and contents & $15(13 \%)$ \\
\hline Cyst & 6 \\
\hline Urinary bladder sludge & 2 \\
\hline Bladder stones & 1 \\
\hline Thickened bladder wall & 1 \\
\hline Thickened colon & 1 \\
\hline Gastric foreign body & 1 \\
\hline Mineralized foci in kidney & 1 \\
\hline Peritoneal effusion & 1 \\
\hline Fluid in uterus & 1 \\
\hline
\end{tabular}

The specific lesion with the highest frequency was splenic nodules $(n=16)$ in the nodular/mass lesions category.

Thirty-eight lesions (35\%) including 6 changes in organ size, 23 nodular or mass lesions, 8 changes in echogenicity and 1 change in architecture or contents were evaluated further by cytology. Many of these demonstrated no cytologic evidence of disease ( $n=11 / 38 ; 29 \%)$. Benign lesions included lymphoid hyperplasia $(n$ $=11 / 38 ; 29 \%$ ), extramedullary hematopoiesis ( $n=$ $8 / 38 ; 21 \%)$ and lipid accumulation in hepatocytes $(n=2 / 38 ; 5 \%)$. In one dog that exhibited peritoneal effusion on abdominal ultrasound, cytology revealed neutrophilic and macrophagic exudate. Metastatic sarcoma was diagnosed in three 
patients. Organs with metastasis were kidney, liver and iliac lymph node. Samples obtained from the liver and a mass adjacent to the bladder were nondiagnostic.

One dog with metastatic sarcoma to the kidneys also had metastasis to the lung. The other two patients with abdominal metastasis did not have evidence of lung or bone metastasis that would have been identified on radiographic examination.

In the studied population, $61 \operatorname{dogs}(52 \%)$ received definitive treatment for OSA, 40 dogs (34\%) received palliative treatment and 17 dogs (14\%) did not receive any specific treatment for OSA. Of the patients that received palliative therapy, 14 dogs ( $n=14 / 40 ; 35 \%)$ had surgery alone, 7 dogs $(n=7 / 40 ; 18 \%)$ had radiation therapy alone, 18 dogs $(n=18 / 40 ; 45 \%)$ had chemotherapy alone, $1 \mathrm{dog}$ received surgery and radiation therapy. Of the 61 dogs that received definitive therapy, 54 ( $n$ $=54 / 61 ; 89 \%)$ received surgery and chemotherapy, $3(n=3 / 61 ; 4.9 \%)$ received definitive irradiation and chemotherapy and $4(n=4 / 61 ; 6.6 \%)$ received surgery, radiation and chemotherapy.

Seventy-nine dogs received some form of chemotherapy. Twenty-two $\operatorname{dogs}(n=22 / 79$; $28 \%)$ received multiple, sequential chemotherapy protocols, while $57(n=57 / 79 ; 72 \%)$ dogs received only one chemotherapy protocol. First line protocols consisted of doxorubicin alternating with carboplatin in 57 dogs, platinum single agent in 13 dogs, and doxorubicin single agent in 9 dogs. Fifteen dogs received one additional protocol, six dogs received two additional protocols, one dog received three additional protocols. Rescue protocols consisted of ifosfamide single agent $(n=12)$, platinum single agent $(n=8)$, doxorubicin single agent $(n=6)$, carboplatin with vinorelbine $(n=1)$, dacarbazine with gemcitabine $(n=1)$, doxorubicin alternating with carboplatin $(n=1)$, and combination of gemcitabine, carboplatin and vinorelbine $(n=1)$. In addition, 16 dogs received pamidronate. Ten dogs $(n=10)$ received pamidronate concurrently with cytotoxic chemotherapy, while six dogs $(n=6)$ received pamidronate alone.

In certain cases, it was clear that ultrasonographic findings influenced treatment decisions. For instance, one patient, who presented with mandibular OSA and had an adrenal mass invading the caudal vena cava, did not pursue surgery for either the adrenal mass or the mandibular OSA, but underwent chemotherapy treatments instead. Similarly, a patient with distal radial OSA with metastasis to the liver underwent palliative radiation in combination with chemotherapy, but later received amputation. In a third case, a patient with distal femoral OSA with metastasis to the iliac lymph node received just palliative radiation without systemic chemotherapy. However in other cases, there appeared to be little alteration in treatment plan. For instance, one patient with distal radial OSA had metastasis to the lungs and kidney underwent amputation followed by chemotherapy. In another case, a patient with scapular OSA that had neutrophilic and macrophagic peritoneal effusion received carprofen $75 \mathrm{mg}$ once daily. The peritoneal effusion resolved after 1 week on carprofen. The patient underwent amputation and later received systemic chemotherapy.

Therefore, to investigate the impact of AUS findings on treatment decisions, we tested for an association between finding any AUS abnormality and whether the animal received definitive therapy, palliative therapy or no therapy. Of the 67 dogs with some sonographic alteration, 8 (12\%) were not treated, 31 (46\%) received palliative therapy and 28 $(42 \%)$ underwent definitive therapy. Whereas in the 51 dogs that had normal AUS, $9(18 \%)$ were not treated, $9(18 \%)$ received palliative therapy, while $33(65 \%)$ received definitive therapy. Treatment choice was statistically associated with AUS findings $\left(\chi^{2}=10.6 ; P=0.005\right)$.

We repeated this analysis using only dogs with appendicular OSA to remove a potential treatment decision bias associated with axial sites. For instance, mandibular OSA is reported to have lower metastatic rate ${ }^{1,6,7}$ and therefore, our definition of definitive therapy might not apply to these cases. In addition, local control is likely more problematic in axial cases, providing another bias to treatment selection. Again, in dogs with appendicular OSA, we observed a statistically significant association between treatment type and identification of a sonographic abnormality $\left(\chi^{2}=7.0 ; P=0.031\right)$. In dogs with appendicular OSA and a sonographic change, $6(11 \%)$ were not treated, $26(46 \%)$ received 
palliative therapy and $24(43 \%)$ received definitive therapy. Whereas in dogs with no sonographic lesion, $6(14 \%)$ were not treated, $9(21 \%)$ received palliative therapy and $28(65 \%)$ received definitive therapy.

At the time of analysis, 73 (62\%) dogs had died of cancer, $4(3 \%)$ were alive and 41 (35\%) were lost to follow-up. The median survival time for these cases was 242 days (95\% CI: 187-298 days). In univariate analysis, age at diagnosis, treatment type and particular imaging findings were associated with outcome (Fig. 1). Older dogs exhibited shorter survival times than younger $\operatorname{dogs}(P=0.048$; Fig. 1A) and dogs receiving definitive treatment lived longer than dogs receiving palliative or no treatment $(P=0.021$; Fig. $1 \mathrm{~B})$. Although the identification of an ultrasonographic abnormality was associated with a shorter survival time, this was not statistically significant $(P=0.071$; Fig. 1C). However, the identification of a sonographic abnormality in either the liver $(n=25 ; P=0.021$; Fig. 1D) or the kidney ( $n=8 ; P=0.003$; Fig. 1E) was statistically associated with shorter survival. Variables that were not statistically associated with survival in this data set included gender, breed, SAP, tumour site (axial versus appendicular), particular types of ultrasound lesions and lesions affecting other abdominal organs ultrasonographically such as the spleen and adrenal glands.

\section{Discussion}

The ability of ultrasonographic imaging to evaluate the internal structure of organs has made it an essential diagnostic tool, replacing abdominal radiography as the first-line choice in the evaluation of the abdomen. ${ }^{3,8-13}$ AUS has been used frequently in the evaluation of newly diagnosed veterinary cancer patients to screen for evidence of metastasis and concurrent conditions that might affect prognosis or treatment. The results of ultrasonographic examination can also serve as a baseline for comparison of patients undergoing treatment. However, despite its routine use, there are few data available to assess the utility of screening veterinary cancer patients using AUS.

In this study population, $2.5 \%(3 / 118)$ of patients with osteosarcoma had metastasis to abdominal organs detected via ultrasound at the time of diagnosis. To the authors' knowledge, this is the first study that reports the frequency of metastasis of OSA to the abdominal organs at initial presentation. In this cohort, $6.0 \%$ of dogs with thoracic radiographs demonstrated metastasis to the lungs at the time of diagnosis, which is consistent with the previous reports demonstrating gross metastatic disease in less than $10-15 \%$ of dogs at presentation. ${ }^{1,2}$ As might be expected for OSA, fewer metastases were found on abdominal ultrasound than thoracic radiographs at the time of diagnosis and one of three cases with abdominal metastasis also demonstrated pulmonary metastasis.

Other imaging techniques used in the evaluation of canine bone tumour patients include bone survey radiography and nuclear scintigraphy. In one study, identification of metastases was more frequent for bone survey radiography $(6.4 \%)$ than for thoracic radiography $(4 \%) .{ }^{1}$ Bone scintigraphy has been used in human and canine osteosarcoma patients as a screening method for detection of bone metastases and for evaluating the primary tumour. Bone scintigraphy has also been used as a predictive indicator of subsequent metastasis in 25 dogs with primary osteosarcoma. ${ }^{14}$ In one study, bone scintigraphy with intravenous injection of HDP labelled with technetium-99m, which is a phosphate analog, $7.8 \%$ detected osseous metastasis in canine osteosarcoma patients on initial presentation. ${ }^{15}$ Given the low number of metastatic cases identified with ultrasonography, this technique is likely far less sensitive than other techniques used for staging dogs with bone tumours.

Although, in this study, few actual metastases were documented using AUS, many alterations were detected by the radiologists performing the studies, including 108 changes in 67 dogs. Of all the ultrasonographic changes described, there were seven lesions $(7 / 108,6.4 \%)$ that were considered clearly capable of altering the prognosis or therapeutic recommendations for the animal. These findings included metastatic sarcoma ( $n$ $=3)$, concurrent malignancy $(n=1)$, peritoneal effusion $(n=1)$, benign splenic masses $(n=1)$ and multiple small stones in the urinary bladder $(n=1)$. 

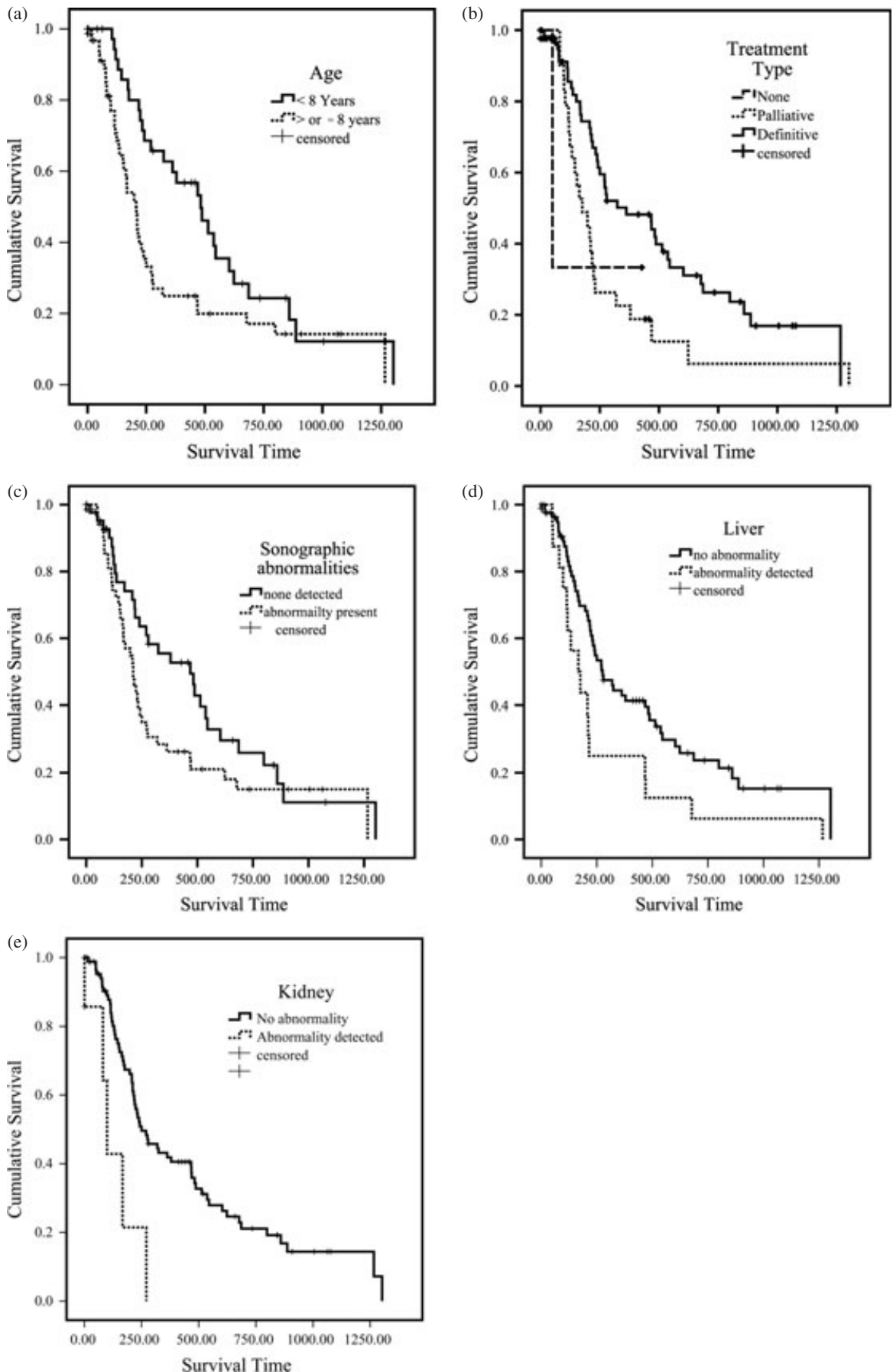

Figure 1. Kaplan-Meier survival curves demonstrating the effect of (A) age, (B) type of treatment, (C) the identification of any ultrasonographic abnormality and identification of ultrasonographic abnormalities affecting the (D) liver or (E) kidney in 118 dogs with osteosarcoma. (A) The median survival times for dogs $<8$ years $(n=47)$ or $\geq 8$ years $(n=71)$ were 482 days and 207 days, respectively $(P=0.048)$. (B) The median survival times for dogs receiving no treatment $(n=17)$, palliative therapy $(n=40)$ or definitive therapy $(n=61)$ were 50,176 and 362 days, respectively $(P=0.021)$. (C) Dogs with and without sonographic abnormalities exhibited median survival times of 211 and 468 days, respectively $(P=0.071)$. (D) Dogs with $(n=25)$ and without $(n=93)$ sonographic abnormalities involving the liver had median survival times of 167 and 276 days, respectively $(P=0.021)$. (E) Dogs with $(n=8)$ and without $(n=110)$ sonographic abnormalities involving the kidney had median survival times of 99 and 250 days, respectively $(P=0.003)$. 
However, of the 108 sonographic changes detected in this study, few received a definitive tissue diagnosis, which underscores the major limitation of abdominal ultrasonographic screening. Ultrasonography is sensitive for lesion detection, but it is not specific for disease aetiology. ${ }^{3}$ Furthermore, definitive evaluation of reported changes is not trivial. In this population, only 38 lesions (35\%) were further evaluated cytologically. Obtaining cells and tissue for more definitive diagnosis can be invasive and is not without potentially serious complications including haemorrhage and tumour dissemination. Furthermore, additional diagnostics to follow-up non-specific ultrasonographic changes can add considerable expense to the pet owner. Thus the difficulty in interpreting ultrasonographic findings can be problematic.

Given the low number of ultrasonographic findings that were considered clearly clinically important in the management of these patients, it is surprising that certain findings were associated with treatment choice and case outcome. In general, dogs with any ultrasonographic alteration fared worse than those with perfectly normal ultrasound exams, though this finding was not statistically significant. Furthermore, lesions in the kidney and liver were associated with a poor outcome. There are numerous possible explanations for this. It is possible that some of these AUS changes actually represented occult metastases. Advanced imaging modalities, such as positron emission tomography may provide a superior means to detect soft tissue metastasis in dogs with bone tumours in the future. Another possibility is that the patients with physical changes in organs involved in drug disposition might not tolerate chemotherapy as well. Finally, it is possible that these findings are influenced not by the specific ultrasonographic finding, but by some confounding variable or simply by chance. One such confounding influence is the impact of owner and clinician with regard to treatment type. Our data suggest that dogs with any sonographic change were significantly more likely to be treated with palliative than definitive therapy. Not surprisingly, dogs that received definitive therapy fared better than dogs that did not receive treatment or received palliative treatment. In most cases, the rationale for the treatment decision was not clear so we are not able to determine whether AUS truly influenced decision making.

Several studies have investigated for prognostic factors in dogs with OSA. In this study, it was not our goal to identify prognostic variables, but to investigate the utility of ultrasonographic imaging. However, in investigating the impact of AUS findings on outcome, we also analysed other standard clinical variables and found that age was associated with outcome. Age is not consistently identified as a prognostic factor for OSA. We found that older dogs in this cohort had shorter survival time than younger dogs $(P=0.048)$, which contrasts with the findings of certain publications. ${ }^{4,16}$ However, these other publications comprised much more uniform populations, including only appendicular cancers that were treated in the same way. Aside from these obvious differences, there may have been other variables such as breed which could influence age distribution. Thus the studies are probably not directly comparable. Similarly, we did not find associations between some variables that have been frequently associated with outcome in canine OSA, such as SAP. Again, these differences likely arise from differences in the population evaluated as well as differences in statistical power for certain variables.

Given the heterogeneity of the population studied, investigating for association between various treatments and outcome, except in the most general sense, was considered inappropriate. In addition, and maybe more importantly, followup frequency and depth of follow-up evaluation varied depending on which type of treatment was selected. For instance, if an owner elected to provide no treatment, the likelihood of having extensive follow-up data for that case was very low. The same is also true for cases receiving palliative treatment. Therefore, cases that received less aggressive treatment also received less followup and are more likely to be censored. It is clear that this biases the survival analysis with respect to treatment. Our finding that dogs fare better when receiving definitive therapy may well reflect the efficacy of treatment, but may also reflect that dogs receiving more intensive treatment and follow-up may be less likely to be euthanized. 
Although this study provides some data for assessing the utility of AUS in managing the canine OSA patient, interpretation of these data should be approached judiciously. The cases involved in this study were managed by several different clinicians and the ultrasound exams were performed by many different sonographers. Thus, a uniform standard for interpretation of ultrasound findings and follow-up of lesions identified is lacking. This is an inherent limitation to a retrospective investigation. Another limitation includes the lack of tissue diagnosis on some of these cases. Forty-one dogs in this study had OSA diagnosis via fine needle aspiration, while six dogs had a presumptive diagnosis of OSA from the radiographic presentation. A study by Britt et al. ${ }^{17}$ showed that ultrasound-guided fine needle aspiration of aggressive bone lesions diagnosed sarcoma with $97 \%$ sensitivity and 100\% sensitivity. While there are other types of primary bone tumours, such as chondrosarcoma, hemangiosarcoma and fibrosarcoma, OSA accounts for up to $85 \%$ of cancer originating in the bone. ${ }^{1,2}$ Therefore, when a diagnosis of sarcoma is obtained via fine needle aspiration, particularly in a characteristic lesion, the possibility of the cancer being OSA is very high.

In conclusion, we found that identification of OSA metastasis at diagnosis with AUS is rare (2.5\%), whereas the identification of some ultrasonographic alteration is common. Although the interpretation of ultrasonographic findings is complicated, our data suggest that some lesions impact treatment decisions and that particular lesions, including those of the kidney and liver, may impact patient outcome.

\section{Acknowledgements}

The author would like to thank Mr. Harm A. Nieuwstadt for his help with figures and reviewing the manuscript and Dr. Candice Bailey for the contribution to the caselist.

\section{References}

1. Dernell W, Ehrhart NP, Straw RC and Vail DM. Tumor of the skeletal system. In: Withrow and MacEwan's Small Animal Clinical Oncology, S Withrow and DM Vail, eds., St. Louis, Saunders Elsevier, 2007.
2. Chun R and de Lorimier LP. Update on the biology and management of canine osteosarcoma. The Veterinary Clinics of North America Small Animal Practice 2003; 33: 491-516.

3. Forrest L. Diagnostic imaging in oncology. In: Withrow and MacEwan's Small Animal Clinical Oncology, S Withrow and DM Vail, eds., St. Louis, Saunders Elvsevier, 2007: 97-111.

4. Spodnick GJ, Berg J, Rand WM, Schelling SH, Couto G, Harvey HJ, Henderson RA, MacEwen G, Mauldin N and McCaw DL. Prognosis for dogs with appendicular osteosarcoma treated by amputation alone: 162 cases (1978-1988). Journal of the American Veterinary Medical Association 1992; 200: 995-999.

5. Hillers KR, Dernell WS, Lafferty MH, Withrow SJ and Lana SE. Incidence and prognostic importance of lymph node metastases in dogs with appendicular osteosarcoma: 228 cases (1986-2003). Journal of the American Veterinary Medical Association 2005; 226: 1364-1367.

6. Straw RC, Powers BE, Klausner J, Henderson RA, Morrison WB, McCaw DL, Harvey HJ, Jacobs RM and Berg RJ. Canine mandibular osteosarcoma: 51 cases (1980-1992). Journal of the American Animal Hospital Association 1996; 32: 257-262.

7. Dickerson ME, Page RL, LaDue TA, Hauck ML, Thrall DE, Stebbins ME and Price GS. Retrospective analysis of axial skeleton osteosarcoma in 22 large-breed dogs. Journal of Veterinary Internal Medicine/American College of Veterinary Internal Medicine 2001; 15: 120-124.

8. Besso JG, Penninck DG and Gliatto JM. Retrospective ultrasonographic evaluation of adrenal lesions in 26 dogs. Veterinary Radiology \& Ultrasound: The Official Journal of the American College of Veterinary Radiology and the International Veterinary Radiology Association 1997; 38: 448-455.

9. Hanson JA, Papageorges M, Girard E, Menard M and Hebert P. Ultrasonographic appearance of splenic disease in 101 cats. Veterinary Radiology \& Ultrasound:The Official Journal of the American College of Veterinary Radiology and the International Veterinary Radiology Association 2001; 42: 441-445.

10. Penninck D, Smyers B, Webster CR, Rand W and Moore AS. Diagnostic value of ultrasonography in differentiating enteritis from intestinal neoplasia in dogs. Veterinary Radiology \& Ultrasound: The Official Journal of the American College of Veterinary Radiology and the International Veterinary Radiology Association 2003; 44: 570-575.

11. Cruz-Arambulo R, Wrigley R and Powers B. Sonographic features of histiocytic neoplasms in the canine abdomen. Veterinary Radiology \& 
Ultrasound: The Official Journal of the American College of Veterinary Radiology and the International Veterinary Radiology Association 2004; 45: 554-558.

12. Cuccovillo A and Lamb CR. Cellular features of sonographic target lesions of the liver and spleen in 21 dogs and a cat. Veterinary Radiology of Ultrasound: The Official Journal of the American College of Veterinary Radiology and the International Veterinary Radiology Association 2002; 43: 275-278.

13. Ramirez S, Douglass JP and Robertson ID. Ultrasonographic features of canine abdominal malignant histiocytosis. Veterinary Radiology \& Ultrasound: The Official Journal of the American College of Veterinary Radiology and the International Veterinary Radiology Association 2002; 43: 167-170.

14. Forrest LJ, Dodge RK, Page RL, Heidner GL, McEntee MC, Novotney CA and Thrall DE. Relationship between quantitative tumor scintigraphy and time to metastasis in dogs with osteosarcoma. Journal of Nuclear Medicine: Official
Publication, Society of Nuclear Medicine 1992; 33: 1542-1547.

15. Jankowski MK, Steyn PF, Lana SE, Dernell WS, Blom CM, Uhrig JL, Lafferty M and Withrow SJ. Nuclear scanning with 99mTc-HDP for the initial evaluation of osseous metastasis in canine osteosarcoma. Veterinary and Comparative Oncology 2003; 1: 152-158.

16. Sottnick JL, Rao S, Lafferty MH, Thamm DH, Morley PS, Withrow SJ and Dow SW. Association of blood monocyte and lymphocyte count and disease-free interval in dogs with osteosarcoma. Journal of Veterinary Internal Medicine 2010; 24: 1439-1444.

17. Britt T, Clifford C, Barger A, Moroff S, Drobatz K, Thacher C and Davis G. Diagnosing appendicular osteosarcoma with ultrasound-guided fine-needle aspiration: 36 cases. The Journal of Small Animal Practice 2007; 48: 145-150. 